\title{
ANALYSIS THE INFLUENCE OF MODULATED AMPLITUDE ON COMMON MODE ERROR BASED ON GPS DATA
}

\author{
Fan Wang ${ }^{1 *}$, Peng Zhang ${ }^{1}$, Zhanyi Sun ${ }^{1}$, Qinglan Zhang ${ }^{1}$, Jin Liu ${ }^{1}$ \\ ${ }^{1}$ National Geomatics Center of China, 28 Lianhuachi West Road, Haidian District, Beijing 100830, China - \\ (wangfan, pzhang, szy, qlzhang,liujin)@ngcc.cn
}

KEY WORDS: GPS, amplitude modulation, time series, common mode error, PCA

\begin{abstract}
:
Time series analysis uses constant amplitude models to estimate seasonal changes, while the actual seasonal changes of station coordinates have varying degrees of modulation. The difference between the real modulation amplitude and the estimated constant amplitude enters the residual sequence. We analysed the contribution of the modulation amplitude to the regional $\mathrm{CME}$ characteristics based on the 410 GPS stations which located in China. The PCA method is used to carry out regional common-mode error analysis on the obtained residuals time series which is after deduction of deformation signals such as tectonic movements. The spectral analysis shows that the CME considering the amplitude modulation significantly weakens the characteristics of the annual cycle. The annual spectral peaks of the north components are reduced by $50 \%$, the east components with a reduction of $80 \%$ and a reduction of $60 \%$ in the elevation component. The results of noise analysis show that the FN in CME that considers amplitude modulation is significantly lower than that of constant amplitude. This indicate that in time series analysis, the 'signal' that has not been estimated due to the oversimplification of the parameters is filtered in the area time will be evolved into CME, which means that CME not only contains errors, but also 'signals', that is, 'signals' that are not correctly modelled will affect the regional filtering effect.
\end{abstract}

\section{INTRODUCTION}

Since the nineties of last century, with the rapid development of GPS technology, a large number of GPS continuous operation reference stations have been deployed around the world. These stations provide important data for geosciences study. However, because of involved many factors in data, errors in the positioning results cannot be totally eliminated. Previous studies have shown that the common mode error is the main source of error in the GPS position time series. It consists of two parts. One part comes from the observation error and the inaccuracy of the analysis model and introduces the station coordinate solution, referred to as the "error" part. For example, an incomplete satellite light pressure model may cause false displacements. Multipath effects at stations, atmospheric delays, ionosphere delays, systematic reference frame errors, etc. are also major sources of error. The other part comes from the signal part that is regarded as the residual error due to the excessive simplification of the model during the analysis of the station coordinate time series, referred to as the "signal" part. For example, the widely used time series analysis only estimates the initial position, linear velocity, co-seismic and post-seismic displacement, and annual and semi-annual terms. Other non-linear deformation signals other than the function attenuation enter the residual sequence. The common part in the residual sequence of the station coordinates is the common mode error (CME). Obviously, the CME also includes the error part and the signal part. One of the signal parts comes from the amplitude modulation of the seasonal changes of the station coordinates. Conventional time series analysis uses constant amplitude models to estimate seasonal changes, while the actual seasonal changes of station coordinates have varying degrees of modulation. The difference between the real modulation ${ }^{1}$ amplitude and the estimated constant amplitude enters the residual sequence. This paper analyses focuses on the analysis of the contribution of the modulation amplitude to the Regional CME characteristics based on the 410 GPS stations which located in China.

\section{METHOD}

We use GAMIT/GLOBK to process the GPS data in order to get the position time series. Then use QOCA to analyse the time series. Both polynomial and spline functions can fit amplitude modulation signals, considering the stations with large amplitude differences, high-order polynomials are needed to characterize them. However, spline function can segment the fitting data, and each segment is fitted with different parameters, which can fully describe the characteristics of different segments, obtain very high-precision fitting results, and easily determine the fitting function. Therefore, this article uses spline function to express the modulation amplitude.

\subsection{GPS DATA PROCESSING METHOD}

At present, GPS data processing methods are basically mature, but many physical models and reference frame involved in data processing are still in the process of continuous improvement and refinement (Petit, 2010). This paper uses GPS double-difference carrier phase observation data, taking every 24 hours as a period, using GAMIT (Herring et al., 2006) software to process and analyze the data. The processing strategies and models used are as follows: (1) Orbital parameters are not constrained tightly. The station positions, satellite orbits (IGS precision ephemeris) and the earth rotation parameters (Bull_A) are allowed to be slightly adjusted, and the radial deviation of the satellite antenna phase center parameters are estimated at the same time; (2)The earth's gravity field, solid tide and extreme tide Models follow the IERS2010 (Petit, 2010) specification; (3)The latest global ocean tide model FES2004

\footnotetext{
* Corresponding author
} 
(Lyard et al., 2006) is used to correct the station displacements caused by the ocean tide, and the change in the solid earth's center of mass caused by the ocean tide is also taken into account. (4) The model calculates the initial values of the dry and wet delay components of the tropospheric zenith distance. At the same time, each station estimates a zenith distance delay correction parameter every 2 hours. The meteorological data are obtained by the GPT2 model (Lagler, 2013). The mapping function adopts the GMF model; (5) Considering the inhomogeneity of the atmosphere, two atmospheric level gradient parameters are added to each station in the east-west and north-south directions.

GAMIT software adopts the double-difference data mode. As the number of stations increases, the CPU calculation time required increases progressively. Therefore, it is not a feasible way to process all reference stations together. According to the geographical distribution, this research divides the base station into 5 subnets in Northeast, Central China, South China, West and Central, respectively, and selects 3-5 common stations as the connection stations between the geographically adjacent subnets. Each subnet there are about $\sim 55$ base stations average. In order to improve the efficiency of data processing, the solution of the global IGS station adopts the solution result of SOPAC (http://sopac.ucsd.edu). The one-day solution gives the coordinates, polar shifts, UT1, satellite orbit parameters, satellite antenna phase centres radial deviation and troposphere zenith delay parameters estimates of each station, and the variance-covariance matrix of these estimates. The single-day relaxation solutions of these subnets are obtained by using the coordinate parameters and satellite orbit parameters of common GPS stations to obtain a joint solution using GLOBK software, thereby obtaining the overall single-day relaxation solution sequence including global IGS stations and my country's reference stations. Then, the seven parameters of similar changes relative to the ITRF2014 ( Altamimi et al., 2016) benchmark are solved by the globally distributed reference stations included in the solution, and the single-day no-benchmark solution is converted to the ITRF2014 framework with the obtained seven parameters, thus obtaining The position solution time series of all reference stations under the ITRF2014 framework.

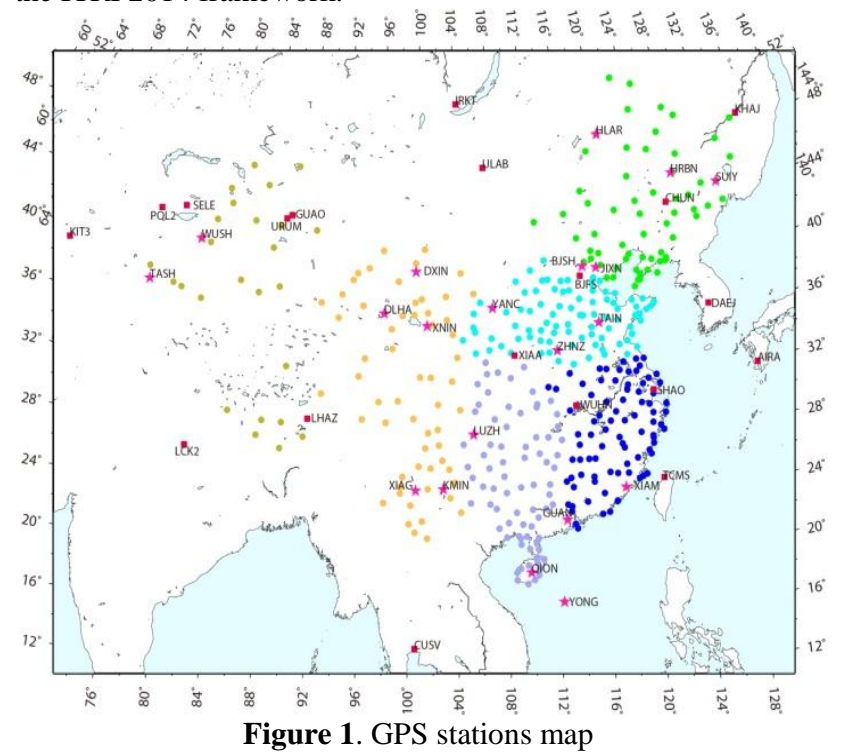

\subsection{TIME SERIES ANALYSES METHOD}

Considering that incomplete time series will affect the final CME results, we filtered the time series of all stations. The principles of filter are: (1) Stations whose time series integrity is less than $80 \%$ are eliminated; (2) The epochs with less than $10 \%$ stations are eliminated, which means that the epochs with less than 41 observation stations at the same day are eliminated. After filtering, the effective stations are 389, the effective epochs are 4225.

The time series of station locations involved long-term tectonic movements, annual and semi-annual seasonal changes, co-seismic and post-seismic displacements caused by major earthquakes, and position mutations introduced by antenna replacement. In addition, scientists have also found the $1.040 \pm$ 0.008 cpy frequency abnormal signal (Collilieux et al. 2007; Ray et al. 2008 ;). Because the period is close to the repetition period of the GPS constellation, Ray et al. call it the GPS "nodal year" signal, and believe that long-period GPS satellite orbit model errors and near-field multipath effects are possible reasons. According to the mixing theory, it needs 25.5 years to accurately separate the 1-year periodic signal from the 1.040-period "intersection year" signal (Davis et al. 2012; Ray et al. 2013). Therefore, this article cannot estimate the "nodal year" signal. First, use parameter estimation to remove these known deformation signals and local effects. The main earthquakes including the Mw9.0 earthquake in Miyagi, Japan earthquake on March 11, 2011, and Lushan Mw7.0 earthquake on April 20, 2013, the Mw 8.1 earthquake in Nepal on April 25, 2015.

\subsection{Modulation amplitudes imulation method}
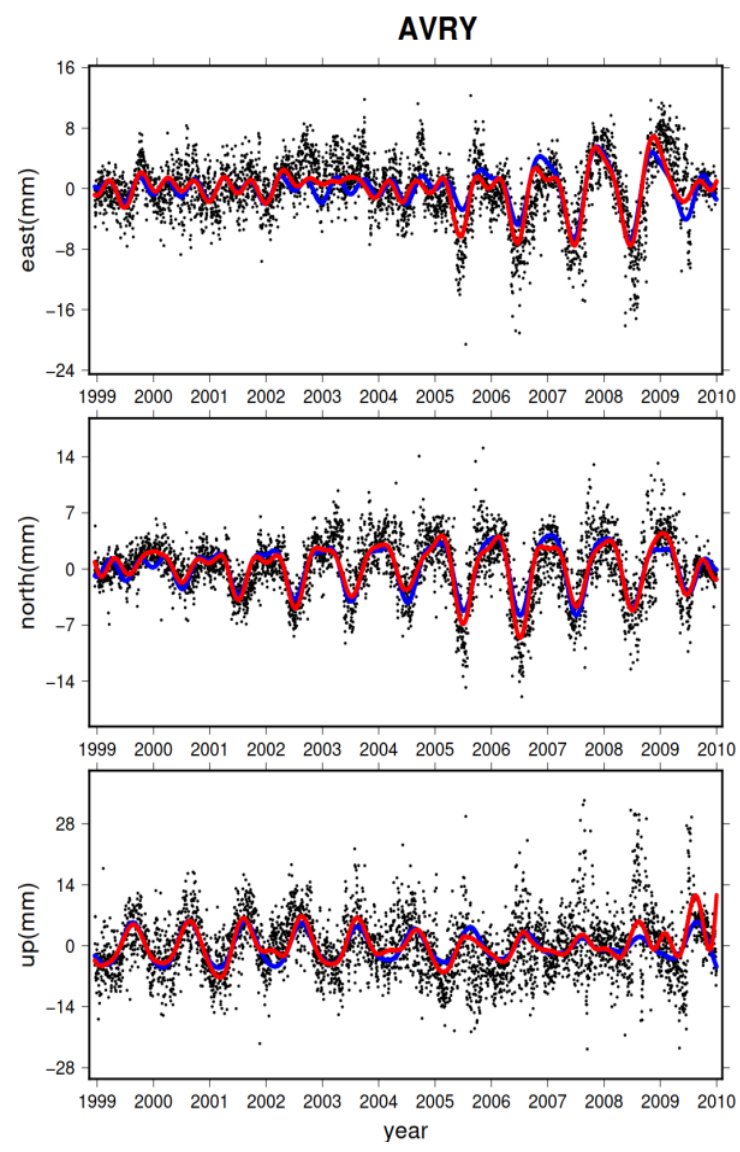

Figure 2. Comparative analysis fitting results of polynomial and spline function. The black dot in the figure represents the residual time series, the blue curve is the polynomial fitting 
result, and the red curve is the spline function fitting result.

In previous studies (Williams et al., 2004; Nikolaidis, 2002; Langbein J. 2008), the annual or half-anniversary constant amplitude and constant phase harmonic models are usually used to express the seasonal signals in the original GPS time series. In fact, climate changes in different years are not consistent, and the response of GPS stations to environmental changes on a seasonal scale is time-varying (Dong et al., 2002). Therefore, there are often these uncompleted and false seasonal signals in the residual series (Freymueller, 2009; Zou et al., 2014). Bennet (2008) assumes that the amplitude of the annual signal in GPS time series changes with time, and uses a semi-parametric model to simulate a "time-varying amplitude period" signal. However, the theory and calculations of this model are very complex and inconvenient for practical applications. Both polynomial and spline functions can be fitted to amplitude modulation signals. We use polynomial and spline functions to fit the amplitude modulation to the residual sequence of the AVRY station (1999-2010) position time series subtracting the structure motion signal and the long-term velocity term respectively. The signals and the fitting results are shown in Figure 1. It can be seen in the figure that for AVRY stations with large amplitude differences in each year, higher-order polynomials are required to characterize them. For example, a 10-order polynomial is used in this article, but the fitting accuracy is not as good as the spline function. This is mainly because the spline function can fit the data For segmentation, each segment is fitted with different parameters, which can fully describe the characteristics of different segments, obtain very high-precision fitting results, and easily determine the fitting function, so this article uses a spline function to express the modulation amplitude.

The first-order spline interpolation function is piecewise linear interpolation, which means the interpolation function is not smooth (it does not have the property of first-order continuous derivative) and cannot match the requirements. Usually the cubic spline interpolation function is often used (Mingfeng, 2016), and the spline function has the second-order continuous derivative characteristic. According to the definition of cubic spline function (Ahlbery et al., 1967), the spline function of the interpolation function on each subinterval is a cubic polynomial, that is, the cubic spline function $\mathrm{S}(\mathrm{x})$ is composed of $\mathrm{n}$ cubic polynomials on $\mathrm{n}$ intervals, and each third-degree polynomial can be described as the following form:

$s_{i}(x)=a_{i} x^{3}+b_{i} x^{2}+c_{i} x+d_{i} \quad i=1,2, \ldots, n$

In this paper, the cubic spline function is used to characterize the amplitude modulation, and the boundary conditions of the spline function value and the first derivative on both sides of the sub-interval are the same. The entire time series is divided into three sub-intervals. The fitting results are shown in the red curve in the figure 2. Due to space limitations, only two stations are given as examples. The jump in the figure in 2011 is the coseismic deformation of the Mw9.0 Miyagi earthquake in Japan. (a) GSLX
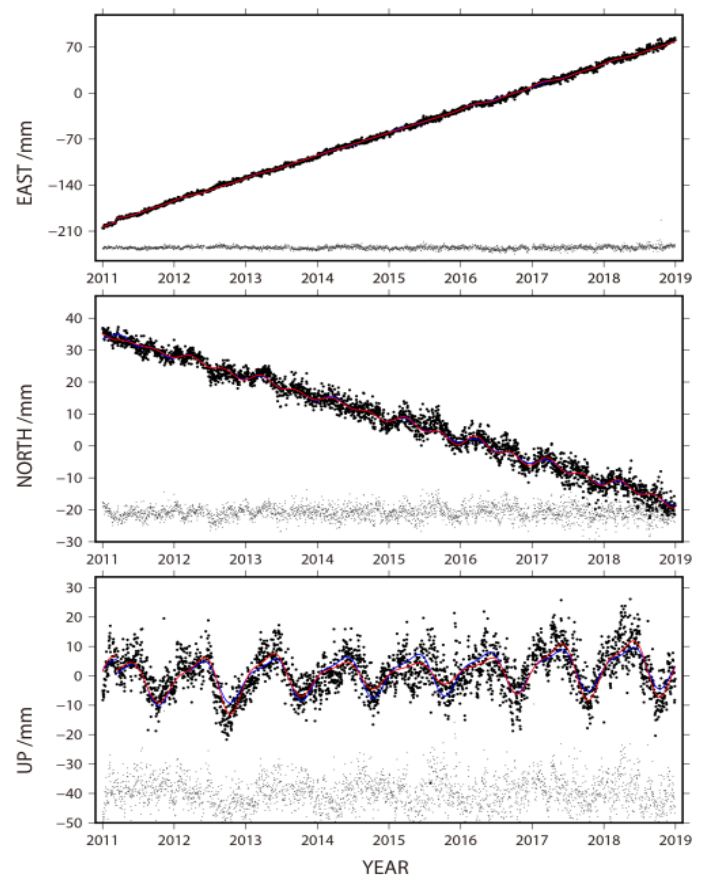

(b) HRBN
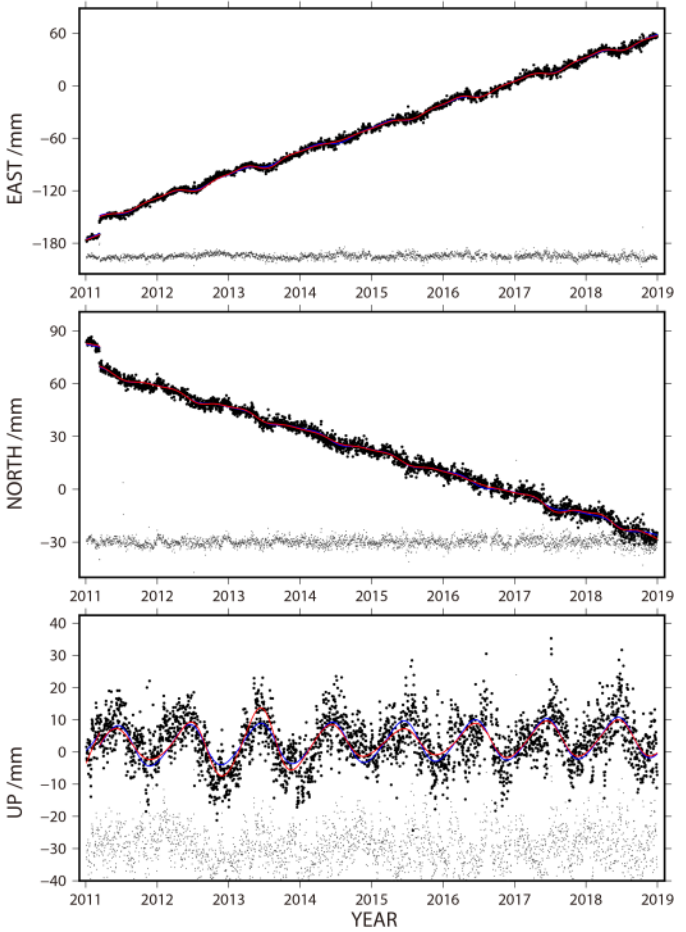

Figure 3. Time series diagram. the black circles represent the time series of the position, blue curve: fit results of the constant amplitude model, red curve: fit results of the amplitude modulation model. The grey circle represents the filtered residual sequence (the residual sequence is shifted for clarity).

\subsection{CME analyse method}

The physical source of the spatially correlated public noise CME in the GPS station's time series remains to be solved, however it can be eliminated by post-processing filtering techniques. The regional filtering method and the PCA method are both based on the GPS coordinate time series. The implicit 
prerequisite of regional filtering is that the spatial response of the regional common mode error is uniformly distributed. The limitation of this simplified assumption makes it difficult to adapt to the precise extraction of the common mode error of the large spatial scale GPS network. The PCA method does not require any prior assumptions, and can directly obtain the CME time series corresponding to each station, and then analyze its spectral characteristics (Dong et al, 2006; Serpelloni et al, 2013), so we used the PCA method to determine the CME.

PCA (Dong et al, 2006) is also called empirical orthogonal function decomposition, which is a decomposition method that constructs an orthogonal basis based on the data itself. If the time series of each station are arranged, each column is one station's time series, which can be expressed as a digital matrix of $\mathrm{m}, \mathrm{x}, \mathrm{n}$ (usually, $\mathrm{m}>\mathrm{n}$ )

$\mathbf{X}=\left(\begin{array}{cccccc}x_{1}\left(t_{1}\right) & x_{2}\left(t_{1}\right) & \ldots & \ldots & x_{n-1}\left(t_{1}\right) & x_{n}\left(t_{1}\right) \\ x_{1}\left(t_{2}\right) & x_{2}\left(t_{2}\right) & \ldots & \ldots & x_{n-1}\left(t_{2}\right) & x_{n}\left(t_{2}\right) \\ \ldots & \ldots & \ldots & \ldots & \ldots & \ldots \\ \ldots & \ldots & \ldots & \ldots & \ldots & \ldots \\ x_{1}\left(t_{m-1}\right) & x_{2}\left(t_{m-1}\right) & \ldots & \ldots & x_{n-1}\left(t_{m-1}\right) & x_{n}\left(t_{m-1}\right) \\ x_{1}\left(t_{m}\right) & x_{2}\left(t_{m}\right) & \ldots & \ldots & x_{n-1}\left(t_{m}\right) & x_{n}\left(t_{m}\right)\end{array}\right)$

Equation (2) can be decomposed by singular value analysis to

$$
\mathbf{X}=\mathbf{U} \Pi \mathbf{V}^{T}
$$

In the formula, $\Pi$ is the diagonal matrix of $m *^{*}$ size, $\mathrm{U}$ is the $(\mathrm{m}$, $\mathrm{x}, \mathrm{m})$ orthogonal normal matrix, also is the $(m, x, n)$ quasi-diagonal matrix; $\mathrm{V}$ is $(n, x, n)$ orthogonal normalized matrix. The most common situation in geosciences research is $\mathrm{m}>\mathrm{n}$, and the rank of $\mathrm{X}$ is $\mathrm{n}$. At this time, the variance matrix of $\mathrm{X}$ can be expressed as

$$
\mathbf{C}=\mathbf{X}^{T} \mathbf{X}=\mathbf{V} \boldsymbol{\Lambda} \mathbf{V}^{T}
$$

In the formula, $\boldsymbol{\Lambda}=\Pi^{T} \Pi$ is $(n, x, n)$ diagonal matrix, and each diagonal element is the power of singular value. At this time, $\mathrm{V}$ constitutes the orthogonal base of $\mathrm{X}$, and $\mathrm{X}$ can be expanded on this orthogonal base to

$$
\mathbf{X}\left(t_{i}, x_{j}\right)=\sum_{k=1}^{n} a_{k}\left(t_{i}\right) v_{k}\left(x_{j}\right)
$$

In the formula, the sequence of expansion coefficients is the $\mathrm{k}$-order principal component, and $\mathrm{vk}(\mathrm{xj})$ is its corresponding site-related spatial response eigenvector, and there is

$$
a_{k}\left(t_{i}\right)=\sum_{j=1}^{n} \mathbf{X}\left(t_{i}, x_{j}\right) v_{k}\left(x_{j}\right)
$$

The PCA method decomposes the time series of the station network solution that changes with time into the principal component in the time domain and the eigenvector in the space domain. Since the decomposition is arranged in accordance with the power (energy) contributed by each principal component, if there is no particularly anomalous local effect of individual stations, the regional common time change characteristics of the station network solution can be based on the first few principal components. The characteristics of change are expressed to the maximum extent, and the characteristic vectors corresponding to these principal components reflect the spatial distribution of the strength of these time changes. Taking into account the large spatial scale of my country's reference station network, this paper uses the PCA method to analyze the time series of my country's reference station, taking the first two principal components to represent the CME.

\section{RESULTS AND DISCUSSIONS}

In order to evaluate the impact of amplitude modulation on CME, this paper also performs PCA analysis on the residual sequence fitted with constant amplitude seasonal terms, and uses spectral analysis and noise analysis to quantify the impact of amplitude modulation on CME.
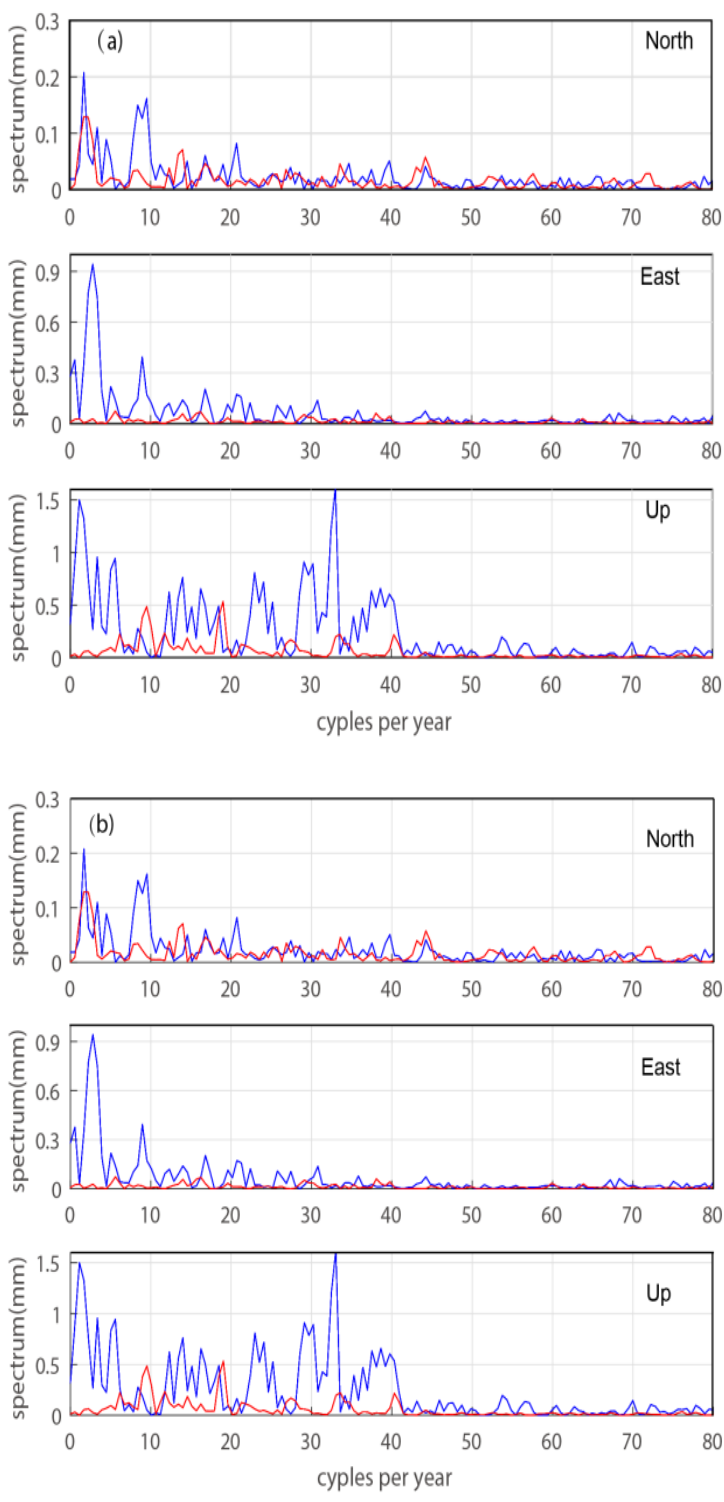

Figure 4. Comparison of CME principal component Spectral analysis considering amplitude Modulation and constant amplitude. Horizontal axis: cycles per year, Blue: constant amplitude, Red: Consider amplitude modulation. (a), the first principal component (b) the second principal component

The spectral analysis (Figure 4) shows that the CME considering the amplitude modulation significantly weakens the characteristics of the annual cycle. The annual spectral peaks of 
the north components are reduced by $50 \%$, the east components with a reduction of $80 \%$ and a reduction of $60 \%$ in the elevation component. This indicates that the time varying characteristics of seasonal terms may be one of the main sources of CME. Precious studies have shown that the residuals of seasonal items such as annuals still exist in the residual sequence of the WN (white noise) + FN (flicker noise) model swap, but the source of this seasonal residual is not clearly pointed out. Our experiments show that the amplitude modulation of the seasonal term is not considered in the time series analysis as the main reason for the residual of the seasonal term.

\begin{tabular}{|c|c|c|c|c|c|}
\hline \multicolumn{3}{|c|}{ items } & $\begin{array}{l}\text { constant } \\
\text { amplitude } \\
/ \mathrm{mm}\end{array}$ & $\begin{array}{l}\text { amplitude } \\
\text { modulation } \\
/ \mathrm{mm}\end{array}$ & $\begin{array}{c}\text { Change } \\
\text { percenta } \\
\text {-ge }\end{array}$ \\
\hline \multirow{6}{*}{$\begin{array}{c}\text { The first } \\
\text { principal } \\
\text { compone } \\
\text {-nt }\end{array}$} & \multirow{3}{*}{$\begin{array}{l}\mathrm{W} \\
\mathrm{N}\end{array}$} & East & $0.7 \pm 0.0$ & $0.6 \pm 0.0$ & $3 \%$ \\
\hline & & North & $0.6 \pm 0.0$ & $0.6 \pm 0.0$ & $1 \%$ \\
\hline & & Up & $1.1 \pm 0.1$ & $1.0 \pm 0.1$ & $6 \%$ \\
\hline & \multirow{3}{*}{$\begin{array}{l}\mathrm{F} \\
\mathrm{N}\end{array}$} & East & $11.8 \pm 0.6$ & $11.0 \pm 0.7$ & $6 \%$ \\
\hline & & North & $8.7 \pm 0.5$ & $8.0 \pm 0.6$ & $9 \%$ \\
\hline & & Up & $29.3 \pm 1.3$ & $27.3 \pm 1.2$ & $7 \%$ \\
\hline \multirow{6}{*}{$\begin{array}{c}\text { The } \\
\text { Second } \\
\text { principal } \\
\text { compone } \\
\text {-nt }\end{array}$} & \multirow{3}{*}{$\begin{array}{l}\mathrm{W} \\
\mathrm{N}\end{array}$} & East & $0.4 \pm 0.0$ & $0.5 \pm 0.0$ & $4 \%$ \\
\hline & & North & $0.4 \pm 0.0$ & $0.6 \pm 0.0$ & $15 \%$ \\
\hline & & Up & $1.2 \pm 0.1$ & $0.7 \pm 0.0$ & $42 \%$ \\
\hline & \multirow{3}{*}{$\mathrm{N}$} & East & $7.7 \pm 0.4$ & $7.5 \pm 0.5$ & $2 \%$ \\
\hline & & North & $7.7 \pm 0.5$ & $7.7 \pm 0.6$ & $1 \%$ \\
\hline & & Up & $21.2 \pm 1.6$ & $14.0 \pm 0.7$ & $34 \%$ \\
\hline
\end{tabular}

Table 1. Noise analysis results.

We also performed noise analysis (Table 1) on the first and second principal component sequences of $\mathrm{CME}$ with modulated amplitude and constant amplitude. The amplitude variation of the first principal component considering the amplitude modulation relative to the constant amplitude is: $\mathrm{WN}$ s increases by $3 \%$ in the east direction, decreases by $1 \%$ in the north direction, decreases by $6 \%$ in the vertical direction, and $\mathrm{FN}$ amplitude decreases by $6 \%$ in the east direction, and decreases by $9 \%$ in the north-south direction, the vertical reduction is $7 \%$. The amplitude variation of the second principal component considering the amplitude modulation relative to the constant amplitude is: WN amplitude increases by $4 \%$ in the east-west direction, $15 \%$ in the north-south direction, $42 \%$ in the vertical direction, and FN amplitude decreases by $2 \%$ in the east-west direction, and increases by $1 \%$ in the north-south direction, the vertical reduction is $34 \%$. It can be seen that after accessing the amplitude modulation, the FN amplitude of the two principal components is significantly reduced compared to the normal amplitude which means the FN in the CME is significantly reduced. It is worth mentioning that $\mathrm{WN}$ and $\mathrm{FN}$ are significantly reduced after estimated the amplitude modulation in the vertical direction, indicating that the characteristics of amplitude modulation in the vertical direction is more obvious. The results of noise analysis show that the FN in CME that considers amplitude modulation is significantly lower than that of constant amplitude. This indicate that in time series analysis, the 'signal' that has not been estimated due to the oversimplification of the parameters is filtered in the area time will be evolved into $\mathrm{CME}$, which means that CME not only contains errors, but also 'signals', that is, 'signals' that are not correctly modelled will affect the regional filtering effect.

\section{ACKNOWLEDGEMENTS}

This work was supported by the National Key Research and Development Program of China (No. 2016YFB0501405) and National Natural Science Foundation of China (No. 41804038) and.

\section{REFERENCES}

Altamimi Z, Rebischung P, Métivier L, et al, 2016. ITRF2014: a new release of the International Terrestrial Reference Frame modeling nonlinear station motions. J Geophys Res Solid Earth, 121(8): 6109 6131.

Bennett R A, 2008. Instantaneous deformation from continuous GPS: contributions from quasi-periodic loads. Geophys J Int, 174(3): 1052 1064.

Collilieux X, Altamimi Z, Coulot D, et al, 2007. Comparison of very long baseline interferometry, GPS, and satellite laser ranging height residuals from ITRF2005 using spectral and correlation methods. J Geophys Res Solid Earth, 112(B12): B12403.

Davis J L, Wernicke B P, Tamisiea M E, 2012. On seasonal signals in geodetic time series. J Geophys Res Solid Earth, 117(B1): B01403.

Dong D, Fang P, Bock Y, et al, 2002. Anatomy of apparent seasonal variations from GPS-derived site position time series. J Geophys Res Solid Earth, 107(B4): ETG 9-1 ETG 9-16.

Dong D, Fang P, Bock Y, et al, 2006. Spatiotemporal filtering using principal component analysis and Karhunen-Loeve expansion approaches for regional GPS network analysis. J. Geophys Res Solid Earth, 111(B3): B03405.

Freymueller J T, 2009. Seasonal position variations and regional reference frame realization. In: Drewes H. Geodetic Reference Frames. Berlin, Heidelberg: Springer, 191 196.

Herring T A, King R W, McClusky S C, 2006. GAMIT reference manual-GPS analysis at MIT, Release 10.3. Cambridge: Massachusetts Institute of Technology.

Lagler K, Schindelegger M, Böhm J, et al, 2013. GPT2: empirical slant delay model for radio space geodetic techniques. Geophys Res Lett, 40(6): 1069 1073.

Langbein J, 2008. Noise in GPS displacement measurements from Southern California and Southern Nevada. J Geophys Res Solid Earth, 113(B5): B05405.

Lyard F, Lefevre F, Letellier T, et al, 2006. Modelling the global ocean tides: modern insights from FES2004. Ocean Dyn, 56(5 6): 394 415.

Ming F, Yang Y X, Zeng A M, et al, 2017. Spatiotemporal filtering for regional GPS network in China using independent component analysis. J Geod, 91(4): 419 440.

Nikolaidis R, 2002. Observation of geodetic and seismic deformation with the Global Positioning System. Ph. D. thesis. San Diego: University of California.

Petit G, Luzum B, 2010. IERS Conventions (2010). IERS Technical Note No. 36, 1 95.

Ray J, Altamimi Z, Collilieux X, et al, 2008. Anomalous harmonics in the spectra of GPS position estimates. GPS Solut, 12(1): 55 64. 
Ray J, Griffiths J, Collilieux X, et al, 2013. Subseasonal GNSS positioning errors. Geophys Res Lett, 40(22): 5854 5860.

Serpelloni E, Faccenna C, Spada G, et al, 2013. Vertical GPS ground motion rates in the Euro-Mediterranean region: new evidence of velocity gradients at different spatial scales along the Nubia-Eurasia plate boundary. J Geophys Res Solid Earth, 118(11): 6003 6024.

Williams S D P, Bock Y, Fang P, et al, 2004. Error analysis of continuous GPS position time series. J Geophys Res Solid Earth, 109(B3): B03412.

Zou R, Freymueller J T, Ding K H, et al, 2014. Evaluating seasonal loading models and their impact on global and regional reference frame alignment. J Geophys Res Solid Earth, 119(2): $1337 \sim 1358$ 